\title{
Patient and Resource Management of Emergency Walk-In Clinic During a COVID-19 Pandemic State Lockdown
}

\author{
Vichar Trivedi' \\ Yasaira Rodriguez Torres ${ }^{2}$ \\ Vaama Patel ${ }^{2}$ \\ Pradeepa Yoganathan (iD) ${ }^{2}$ \\ 'Wayne State University School of \\ Medicine, Detroit, MI, USA; ${ }^{2}$ Department \\ of Ophthalmology, Kresge Eye Institute, \\ Wayne State University School of \\ Medicine, Detroit, MI, USA
}

Objective: To gather data on the most common chief complaints, diagnoses, in-office procedures, and surgeries that presented to the Kresge Eye Institute's Emergency Clinic in Detroit, Michigan during the COVID-19 state lockdown period to provide data for staff and resource allocation in future waves.

Design: Retrospective study.

Participants: All patients 18 years or older presenting for ophthalmic consultation to the Kresge Eye Institute Emergency Walk-in Clinic between March 23rd and April 17th, 2020 were included in the study.

Methods: All patients who met the inclusion criteria were indexed based on their initial encounter date and patients were stratified by urgent and non-urgent ophthalmic reasons for evaluation. Patient demographics, medical history, chief complaint, diagnosis, and need for surgical intervention and procedures were obtained from the electronic medical records.

Results: The most common diagnoses were corneal disease (31.4\%), followed by vitreoretinal disease $(25.3 \%)$, orbit-eyelid pathology $(8.8 \%)$, and glaucoma-related issues $(8.8 \%)$. The most common office procedure was intravitreal injections $(37.5 \%)$ followed by foreign body removal (21.9\%), and pan-retinal photocoagulation laser (21.9\%). Retina surgery was the most common emergency surgery representing $73.3 \%$ of the total, the second most common was keratoplasty $(13 \%)$.

Conclusion: Future implementation of protocols for triaging based on chief complaints can aid in protecting patients and expanding the role of distanced assessment with telemedicine. Suggested management of an emergent clinic requires availability of retina and cornea specialists given the majority of visits, procedures, and surgeries were related to their area of expertise.

Keywords: retina, cornea, COVID 19, SARS-CoV-2 infection, COVID-19 pandemic, ophthalmology, clinical protocol, coronavirus disease-19

\section{Introduction}

Severe acute respiratory syndrome coronavirus 2 (SARS-CoV-2) emerged in late 2019, leading to the worldwide COVID-19 pandemic. Several investigations have shown transmission via respiratory droplets, airborne, and close contact. ${ }^{1-3}$ These facts put ophthalmologists and their patients at a higher risk of contagion during patient examination. Many ophthalmology practices also serve populations that include older patients with multiple comorbid conditions that are more prone to infection and severe complications. ${ }^{4}$
Correspondence: Pradeepa Yoganathan Department of Ophthalmology, Kresge Eye Institute, Wayne State University School of Medicine, 4717 St. Antoine Street, Detroit, MI, 4820I, USA

Email deepayoganathan@gmail.com 
Given these risks federal, state officials, and the American Academy of Ophthalmology issued statements recommending all ophthalmologists to cease providing any treatment other than urgent or emergent care. ${ }^{5}$ Their guidelines outlined general recommendations, but individual clinics decided the specifics of protocol implementation, staff-resource allocation, environmental control, and the use of technology such as telehealth. Clinic management during the pandemic required adequate COVID screening, creating and adopting protocols for urgent patients, guidelines on triaging based on chief complaints, and reconstruction of patient access and flow in the clinic. ${ }^{3,4}$ To reduce transmission practitioners practiced thorough hand washing, disinfection of patient areas and instruments, social distancing, use of protective barriers, masks, and gowns during examination of at-risk patients. ${ }^{3,4}$ Also all patients were advised to forgo coming to health care centers for non-urgent evaluations. ${ }^{4}$ Progressive harm to a patient's vision with delayed treatment was the driving factor for urgent evaluation during this time. ${ }^{4}$

The purpose of this paper is to describe our emergency walk-in clinic at the Kresge Eye Institute (KEI) that delivered care during the COVID -19 lockdown period. We report the most common chief complaints, diagnoses, procedures, and surgeries that presented during this time to provide data for protocol implementation and staff-resource allocation. Our institute is a tertiary referral center located in Detroit, Michigan. From March 24 to June 8, 2020, the state of Michigan enforced a stay at home order to restrict the spread of the virus. ${ }^{6,7}$ We serve a population that is $78.6 \%$ African American, with a median household income of $\$ 29,481$, and $14.6 \%$ of the population has an education of a bachelor's degree or higher. ${ }^{8}$ Communities with poor social determinants of health have a higher risk of medical complications. ${ }^{9}$ We propose guidelines for patient screening based on chief complaints and clinic management in an underserved community that maximizes resource utilization during future lockdowns due to the resurgence of COVID-19 cases.

\section{Measures During the COVID-I 9 Lockdown}

Measures taken for COVID-19 precaution listed in Table $1 .^{5}$

Table I Measures Taken During the COVID-19 Pandemic State Lockdown

\begin{tabular}{|c|c|}
\hline $\begin{array}{l}\text { Administrative and resource } \\
\text { management }\end{array}$ & $\begin{array}{l}\text { - Cessation of non-emergent services and elective surgeries to decrease clinic volume } \\
\text { - Contact patients via phone call to cancel or reschedule clinic appointments }\end{array}$ \\
\hline Environmental control & $\begin{array}{l}\text {-All patient visits took place in the first-floor clinics } \\
\text {-All people entering the building were funneled through one entry point } \\
\text {-All were required to sanitize hands with ethyl alcohol } 70 \% \text { upon entry } \\
\text { - Chairs in the common areas were spaced } 6 \text { feet apart } \\
\text { - Patients were evaluated individually, no companions allowed to come unless required } \\
\text {-Isolation rooms were designated for the evaluation of COVID- } 19 \text { suspected or confirmed positive cases } \\
\text { - Transparent protective shields were installed on slit lamps } \\
\text { - Exam rooms were disinfected using diluted sodium hypochlorite after each patient } \\
\text {-Equipment such as slit lamps was disinfected using } 70 \% \text { ethyl alcohol after each use } \\
\text { - Tonometry with disposable covers was used to measure intraocular pressure }\end{array}$ \\
\hline Screening and Triaging & $\begin{array}{l}\text {-All were required to complete a COVID-19 symptom questionnaire before entering the clinical area. } \\
\text {-Those positively screened for COVID-19 symptoms without an urgent ophthalmic complaint were directed to } \\
\text { go home and contact their primary care provider or seek care at the nearest emergency department } \\
\text {-Triaging was in accordance with the published guidelines by the American Academy of Ophthalmology for } \\
\text { urgent and non-urgent reasons for evaluation } \\
\text {-Non-urgent cases encouraged to reschedule the appointment for a later date } \\
\text {-Urgent cases proceeded to an exam room for evaluation }\end{array}$ \\
\hline $\begin{array}{l}\text { Staff Protection and Personal } \\
\text { Protective Equipment }\end{array}$ & $\begin{array}{l}\text { - Healthcare workers were instructed to wear a surgical mask or N95 masks at all times. } \\
\text { - The evaluating physician was encouraged to use masks, gloves, and eye protection for all encounters } \\
\text { - Patients were encouraged to wear masks } \\
\text { - Urgent cases that were positive on COVID- } 19 \text { screening were immediately placed in the designated isolation } \\
\text { rooms. Evaluating ophthalmologist was recommended to wear an N95 mask, gown, gloves, and eye protection }\end{array}$ \\
\hline
\end{tabular}


Table 2 Demographic Information

\begin{tabular}{|l|l|l|l|l|l|}
\hline Age & Range: $18-94$ yo & Min: 18 yo & Max: 94 yo & \multicolumn{3}{|l|}{ Mean: 54.2 yo } \\
\hline Sex & Male: $42.8 \%$ & Female $57.2 \%$ & & & \\
\hline Race & African: $73.8 \%$ & White $15.7 \%$ & Other: $10.5 \%$ & COPD:7.0\% & CHD:6.6\% \\
\hline Comorbidities & HTN: $53.7 \%$ & Diabetes: $29.3 \%$ & KD:8.3\% \\
\hline
\end{tabular}

Note: Other: Asian, Latino, Middle Eastern, Native Hawaiian, patients that did not disclose.

Abbreviations: HTN, hypertension; KD, kidney disease; CHD, coronary heart disease; COPD, chronic obstructive pulmonary disease; yo, years old.

\section{Methods}

All adult patients presenting for ophthalmic consultation to KEI between March 23, 2020, and April 17, 2020, were included in this study. The inclusion criteria included all patients age 18 years and older who presented during the study dates. This study was approved by the Wayne State University Institutional Review Board (IRB:20-04-2136) and consent was waived due to its retrospective study design. Patient demographics, medical history, chief complaint, diagnoses, and need for surgical intervention were obtained from medical records. All patients were indexed based on their initial encounter date, and patients were stratified by urgent and non-urgent ophthalmic reasons for evaluation. All chief complaints were categorized into four groups: follow up/post-op, vision change, painirritation-itching, and external adnexa issues. All diagnoses made during the patient visit were further stratified into groups by the primary anatomic region affected. The groups included cornea, orbit/eyelid, vitreoretinal, lens, uveitis, glaucoma, trauma, and neuro-ophthalmology.

All statistics were completed using IBM SPSS Statistics for Windows, version 26 (IBM Corp., Armonk, N.Y., USA), and all tables and figures were generated in Excel version 16.27 (Microsoft Corp, Washington). A comparison of all variables was conducted between urgent and non-urgent ophthalmic consultations. Analysis of the statistical significance of variables between cohorts was done using an odds ratio for categorical variables and a Spearman correlation for continuous variables. All analyses were conducted as two-tailed tests with a significance level of $\mathrm{p}<0.05$.

\section{Results}

A total of 320 visits to KEI from 229 patients met the inclusion criteria for the study. The majority of the patients who presented were African American (73.8\%) with a mean age of $54.2 \pm$ SD 17.1 years. Females $(57.2 \%)$ represented a higher proportion of participants. Of the co- morbidities, hypertension (53.7\%) was the most prevalent, and a significant minority also had diabetes (29.3\%) (Table 2). Of the total visits, $49.1 \%$ were for an acute chief complaint, and $37.5 \%$ were for a follow-up visit. Of the 229 patients seeking care, $70.3 \%$ had an urgent condition and required a full exam. Patients who presented with a chief complaint of vision changes and operative related visits were likely to be evaluated as urgent exams with an $\mathrm{OR}=0.401$ (95\% CI [0.214-0.755], $\mathrm{p}=0.0046)$ and $\mathrm{OR}=3.510 \quad(95 \%$ CI [1.779-6.924], $\mathrm{p}=0.0003)$, respectively. Patients who presented with a chief complaint of pain, irritation, itching, and adnexal issues were likely to be classified as non-urgent exams with an $\mathrm{OR}=1.094(95 \%$ CI [0.611-1.957], $\mathrm{p}=0.7627)$ and $\mathrm{OR}=0.173$ (95\% CI [0.092-0.328], $\mathrm{p}<0.0001$ ), respectively (Table 3 ). The most common diagnosis overall was vitreous hemorrhage (4.4\%) followed by, corneal ulcer (3.9\%), and retinal detachment (3.9\%) (Table 4). When the diagnosis was divided into anatomical categories, corneal disease (31.4\%) was the most common, followed by vitreoretinal diseases $(25.3 \%)$, orbit-eyelid pathology $(8.8 \%)$, and glaucoma-related issues (8.8\%) (Table 5).

Thirty-two patients required an urgent office procedure and 15 patients an emergency surgery. The most common procedure was intravitreal injection (37.5\%), followed by foreign body removal $(21.9 \%)$, and pan-retinal photocoagulation laser (21.9\%) (Table 6). Retina surgery was the most common representing $73.3 \%$ of the total followed by keratoplasty (13\%) (Table 7).

Table 3 Chief Complaints

\begin{tabular}{|l|r|r|}
\hline Chief Complaint & Frequency & Percent \\
\hline Acute vision change & 90 & $39.3 \%$ \\
\hline Acute pain-irritation-itching & 91 & $39.7 \%$ \\
\hline Post-operative examination & 86 & $37.5 \%$ \\
\hline Acute external adnexa issue & 60 & $26.2 \%$ \\
\hline
\end{tabular}


Table 4 Most Common Diagnoses

\begin{tabular}{|c|c|c|}
\hline & & Frequency \\
\hline \multirow[t]{16}{*}{ Diagnosis } & Vitreous hemorrhage & 10 \\
\hline & Corneal ulcer & 9 \\
\hline & Retinal detachment & 9 \\
\hline & Primary open angle glaucoma & 8 \\
\hline & Chalazion & 8 \\
\hline & Corneal abrasion & 8 \\
\hline & $\begin{array}{l}\text { Foreign body of the cornea } \\
\text { Dry eye }\end{array}$ & $\begin{array}{l}8 \\
7\end{array}$ \\
\hline & Viral conjunctivitis & 7 \\
\hline & Ruptured globe & 6 \\
\hline & Age related macular degeneration & 5 \\
\hline & Traumatic iritis & 4 \\
\hline & Proliferative diabetic retinopathy & 4 \\
\hline & Posterior vitreous detachment & 4 \\
\hline & Vitreous floaters & 4 \\
\hline & Nuclear sclerosis & 4 \\
\hline & Age related cataract & 4 \\
\hline
\end{tabular}

Table 5 Diagnosis: Category of Eye

\begin{tabular}{|c|c|c|c|}
\hline & & Frequency & Percent \\
\hline \multirow[t]{9}{*}{ Category } & Cornea & 61 & 31.4 \\
\hline & Vitreo-retinal disease & 49 & 25.3 \\
\hline & Orbit/eyelid & 17 & 8.8 \\
\hline & Glaucoma & 17 & 8.8 \\
\hline & Lens & 16 & 8.2 \\
\hline & Uveitis & 16 & 8.2 \\
\hline & Trauma & 11 & 5.7 \\
\hline & Neuro-ophthalmology & 7 & 3.6 \\
\hline & Total & 194 & 100.0 \\
\hline
\end{tabular}

A comparison of the total patient flow coming into the clinic with the total SARS-CoV-2 positive case counts in the city of Detroit was performed. Figure 1 shows a graphical representation of this data. The total positive case counts in Detroit during the time of this study to the end of week 1, week 2, week 3, and week 4 were 1772 , 4072,5537 , and 6530 , respectively. The total weekly case
Table 6 Office Procedures

\begin{tabular}{|l|l|r|r|}
\hline \multicolumn{2}{|c|}{ Type } & Frequency & Percent \\
\cline { 2 - 4 } & Anti-VEGF & 12 & 37.5 \\
\cline { 2 - 4 } & Foreign Body Removal & 7 & 21.9 \\
\cline { 2 - 4 } & Pan-retinal Photocoagulation & 7 & 21.9 \\
\cline { 2 - 4 } & Placement of bandage contact lens & 2 & 6.3 \\
\cline { 2 - 4 } & Corneal culture and plate & 1 & 3.1 \\
\cline { 2 - 4 } & Epilation & 1 & 3.1 \\
\cline { 2 - 4 } & Bleb needle revision & 1 & 3.1 \\
\cline { 2 - 4 } & Suture removal & 1 & 3.1 \\
\cline { 2 - 4 } & Total & 32 & 100.0 \\
\hline
\end{tabular}

Table 7 Surgical Procedure

\begin{tabular}{|c|c|c|c|}
\hline & & Frequency & Percent \\
\hline Type & Vitreo-retinal surgery* & II & $73.3 \%$ \\
\hline & Keratoplasty & 2 & $13.3 \%$ \\
\hline & Ruptured globe repair & I & $6.7 \%$ \\
\hline & Temporal artery biopsy & $\mathrm{I}$ & $6.7 \%$ \\
\hline & Total & 15 & 100.0 \\
\hline
\end{tabular}

Note: *Pars plana vitrectomy, membrane peel, endolaser, C3F8 gas, scleral buckling, silicon oil, retisert.

count at the clinic at the previously mentioned time points were 91, 79, 78, and 72, respectively (Spearman's $\mathrm{r}=$ $-0.356 ; \mathrm{p}=0.063)$. The number of new cases per week recorded at the clinic during this time, at 78, 55, 50, and 46 , respectively (Spearman's $r=-0.448 ; \mathrm{p}=0.017$ ). When examining the rates of follow up weekly, the visits changed from 54, 35, 42, and 32, respectively (Spearman's r= $-0.356 ; \mathrm{p}=0.063$ ) (Table 8).

\section{Discussion}

The chief complaints, diagnoses, surgeries, and procedures outlined in this paper provide insight into the operation of an emergency clinic during the COVID-19 pandemic. Our data describes the emergency assessment protocol and diagnostic chief complaints that warranted declining stayat-home recommendations. Cornea and retina subspecialists need to be available given that the majority of urgent visits, procedures, and surgeries were related to their area of expertise. Anti-VEGF injections were the most common 


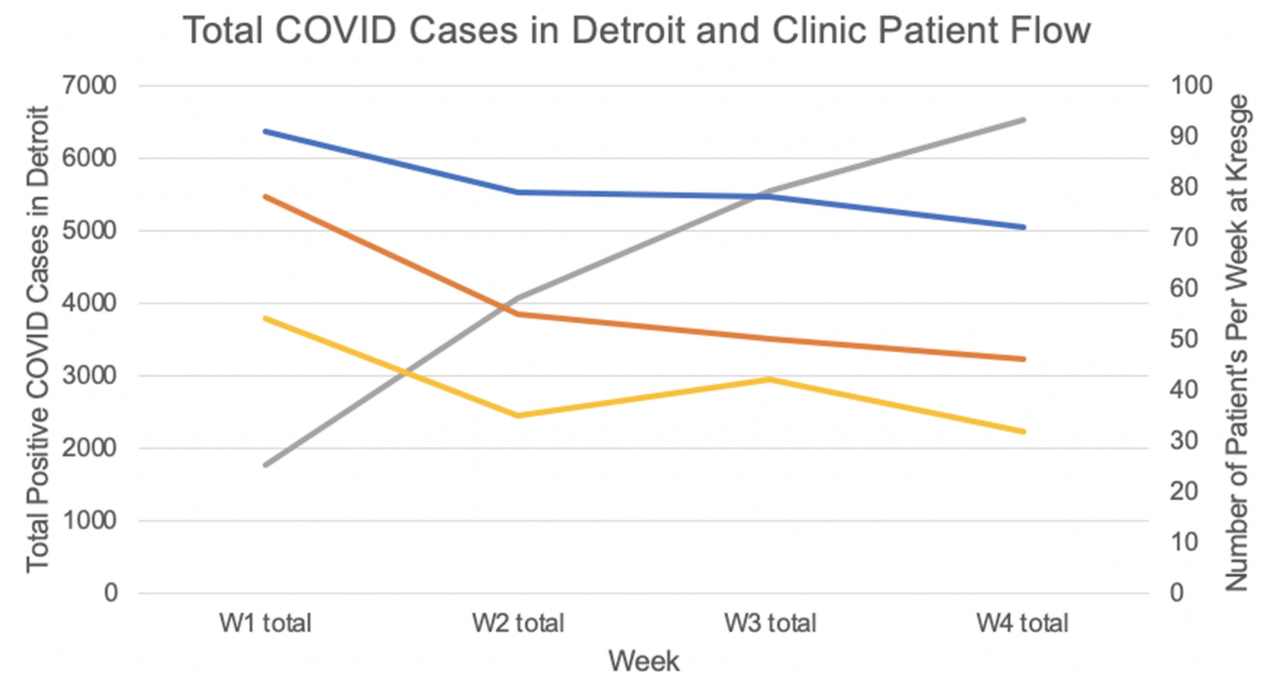

Detroit: Total Cases Clinic: All Visits Clinic: First Cases $\quad$ Clinic: Follow Up

Figure I The trend of the total positive COVID case counts in Detroit, Michigan during the period of this study as well as patient flow into Kresge during this time. The grey line shows the total COVID cases in Detroit during the study period. The blue line represents all visits to the clinic per week during the study period. The orange line represents the patient's first visits during the study period. The yellow line represents all patients presenting for follow-up appointments.

procedure; thus, there must be an adequate supply of the medication and materials used for its administration. These key findings can aid offices in allocating resources, workforce, and clinician expertise. Many experts predict that COVID-19 may lead to multiple waves of positive cases. Therefore, offices must prepare for both short and longterm impacts.

Of the 229 patients who presented $29.7 \%$ had non-urgent complaints. The complaints of pain, irritation, itching, and adnexal issues were likely to be classified as non-urgent exams. Benign pathologies such as dry eye and chalazion still motivated patients to seek evaluation at the emergency clinic during lockdown. These issues could provide an outlet to utilize remote screening methods to reduce the risk of contagion.

Understanding the correlation between complaints and urgent exams can be advantageous for the implementation of teleophthalmology. Specific complaints and symptoms seemed to warrant urgent examination and intervention.

Table 8 Correlation Between Positive COVID Cases in Detroit and Clinic Visits

\begin{tabular}{|l|r|r|}
\hline Type of Clinic Visit & r Value & p value \\
\hline Total cases per week & -0.356 & 0.063 \\
\hline New cases per week & -0.448 & 0.017 \\
\hline Follow-up cases per week & -0.356 & 0.063 \\
\hline
\end{tabular}

The use of teleophthalmology increases access while minimizing risk, and it can be a useful adjunct for low-risk follow-ups and external adnexal pathology. ${ }^{10}$ Yet, until a more thorough system of remote evaluation develops, many complaints will necessitate full in-person workup. Furthermore, many centers, such as ours, serve an underserved population that has limited access to technology. Such outlets have the potential to widen health care disparities. In these circumstances, phone triaging may have limited utility. Therefore, having a transparent in-person protocol has great value.

Evaluation of trends during the weeks the pandemic peaked provides insight into the patients' care-seeking behavior. We noted a decrease in the number of patients presenting for acute complaints as the number of COVID19 positive cases rose. Moon et al looked at visits in an ophthalmic emergency department at Massachusetts Eye and Ear during the COVID-19 lockdown period and noted a significant downward trend in visits as compared to the two preceding years. ${ }^{11}$ Showing that the decreases noted could be as a result of government restrictions. Conversely, in our study follow-ups remained relatively stable, especially in the middle of the study. These trends possibly correlate with the risk-benefit ratio for a patient. The stable follow-up rate may reflect comfort with the staff and environmental control provided. Understanding such trends is crucial for meeting evolving patient expectations. As we transition back to routine care, mitigation 
strategies will need to be integrated as part of a new normal. These strategies will minimize risk and build patient rapport. Despite a resurgence of cases, patients may be more likely to seek timely care.

Other papers coming out since the start of the pandemic have stressed the importance of protocol measures with emphasis on mitigation strategies involving environmental control, patient flow, the use of protective equipment, barriers, proactive handwashing, and disinfection of all equipment used. ${ }^{3,4}$ Studies such as the one done by Moon et al showed a decrease in visits as government restrictions were put into place. ${ }^{11}$ The authors noted the volume of non-urgent and urgent visits both fell during this time but the non-urgent visits decreased at a much greater proportion as compared to urgent visits. ${ }^{11}$ The authors also noted an increase in the proportion of urgent surgical interventions during this time. ${ }^{11}$ This study did not exclude patients who presented to the ED on multiple occasions from their data, our study made a distinction between patients and visits so that there would be no confounding for patients who showed up frequently.

This retrospective study has limitations. Data was collected from the EMR; thus, the data is dependent on accurate record-keeping. Although relevant for an urban clinical setting, these results may not hold for other settings. Despite these limitations, our study has several strengths. Our sample size is large, and the patients who arrived at our walk-in clinic reflect issues managed by urban centers. The data in this paper reflects the issues our patient population deemed important during the lockdown period.

Given the absence of validated SARS-CoV-2 therapies, there is a high likelihood of future government lockdowns and social restrictions. Our measures allowed us to deliver emergent patient care in a safe clinical environment. We share our experience with common chief complaints, diagnoses, procedures, and surgeries. Our study can serve as a framework for clinics to prepare for future case waves and lockdowns. Interesting directions for future investigation include how the number of clinic visits per week will change in the subsequent waves of the pandemic. In addition to whether the patients present with a similar set of complaints in the second wave, and if they are comfortable with the mitigation strategies put forth by healthcare providers.

\section{Conclusion}

The beginning of the COVID pandemic led to the unprecedented shut down of many valuable services, including ophthalmology clinic visits and elective surgeries. Our paper aims to tease the nature of an emergent walk-in ophthalmologic clinic, identifying symptoms patients deem as significant versus diseases and procedures that required urgent follow-up. This includes chief complaints related to corneal $(31.4 \%)$ and vitreoretinal disease $(25.3 \%)$, urgent cases requiring retinal surgery (73.3\%), and procedures such as intravitreal injections $(37.5 \%)$. This data can help aid in building protocols that cater to such needs. Should the state government mandate lockdown again, this paper can serve as a guide for other ophthalmology clinics in resource management for various subspecialties and implementation of safe and effective protocols.

\section{Data Sharing Statement}

The data that support the findings of this study are available on request from the corresponding author.

\section{Ethical Approval}

Wayne State University Institutional Review Board waived consent for this study (IRB:20-04-2136) due to data being gathered from retrospective chart review. The authors certify that this work is HIPAA compliant and adhered to the tenets of the Declaration of Helsinki. The privacy of all participants was respected, and data was anonymized and maintained with confidentiality.

\section{Author Contributions}

All authors made substantial contributions to conception and design, acquisition of data, or analysis and interpretation of data; took part in drafting the article or revising it critically for important intellectual content; agreed to submit to the current journal; gave final approval of the version to be published; and agree to be accountable for all aspects of the work.

\section{Funding}

This research received no specific grant from any funding agency in the public, commercial, or not-for-profit sectors.

\section{Disclosure}

The authors report no potential conflicts of interest for this work.

\section{References}

1. Jin Y, Yang H, Ji W, et al. Virology, epidemiology, pathogenesis, and control of COVID-19. Viruses. 2020;12(4):372. doi:10.3390/v12040372 
2. Setti L, Passarini F, De Gennaro G, et al. Airborne transmission route of COVID-19: why 2 meters/ 6 feet of inter-personal distance could not be enough. Int J Environ Res Public Health. 2020;17(8):2932. doi:10.3390/ijerph17082932

3. Pei X, Jiao X, Lu D, Qi D, Huang S, Li Z. How to face COVID-19 in ophthalmology practice. Med Hypothesis Discov Innov Ophthalmol. 2020;9(3):164-171.

4. $\mathrm{Hu} \mathrm{VH}$, Watts E, Burton $\mathrm{M}$, et al. Protecting yourself and your patients from COVID-19 in eye care. Community Eye Health. 2020;33(108):S1-s6.

5. Ophthalmology AAO. Important coronavirus updates for ophthalmologists. News Web site; 2020. Available from: https://www. aao.org/headline/alert-important-coronavirus-context. Accessed April 20, 2020.

6. Whitmer G. Executive order 2020-21 (COVID-19) - rescinded; 2020. Available from: https://www.michigan.gov/whitmer/0,9309,7-38790499_90705-522626-,00.html. Accessed July 4, 2020.
7. Whitmer G. Executive order 2020-110 (COVID-19) (June 1, 2020); 2020. https://www.michigan.gov/whitmer/0,9309,7-387-90499_ 90705-530620-,00.html. Accessed July 4, 2020.

8. Bureau USC. Detroit city, Michigan; 2019. Available from: https:// www.census.gov/quickfacts/fact/table/detroitcitymichigan,MI/ PST045219. Accessed June 25, 2020.

9. Braveman P, Egerter S, Williams DR. The social determinants of health: coming of age. Annu Rev Public Health. 2011;32(1):381-398. doi:10.1146/annurev-publhealth-031210-101218

10. Chen RWS, Abazari A, Dhar S, et al. Living with COVID-19: a perspective from new york area ophthalmology residency program directors at the epicenter of the pandemic. Ophthalmology. 2020;127 (8):e47-e48. doi:10.1016/j.ophtha.2020.05.006

11. Moon JY, Miller JB, Katz R, et al. The impact of the COVID-19 pandemic on ophthalmic care at an eye-specific emergency department in an outbreak hotspot. Clin Ophthalmol. 2020;14:4155-4163. doi:10.2147/OPTH.S285223
Clinical Ophthalmology

\section{Publish your work in this journal}

Clinical Ophthalmology is an international, peer-reviewed journal covering all subspecialties within ophthalmology. Key topics include: Optometry; Visual science; Pharmacology and drug therapy in eye diseases; Basic Sciences; Primary and Secondary eye care; Patient Safety and Quality of Care Improvements. This journal is indexed on PubMed

Submit your manuscript here: https://www.dovepress.com/clinical-ophthalmology-journal
Dovepress

Central and CAS, and is the official journal of The Society of Clinical Ophthalmology (SCO). The manuscript management system is completely online and includes a very quick and fair peer-review system, which is all easy to use. Visit http://www.dovepress.com/ testimonials.php to read real quotes from published authors. 